\title{
The role and practice of the genetic nurse: report of the AGNC Working Party
}

\author{
H Skirton, C Barnes, G Curtis, J Walford-Moore
}

\begin{abstract}
The role of the genetic nurse has evolved historically with the emergence of clinical genetics in the field of health care. During 1994, a Practice Working Party was convened by the Genetic Nurses and Social Workers Association in response to discussion about the role of the nurse within and between regional genetics centres. The Working Party conducted a study of the current nursing practice and attitudes of nurses and clinicians to the nursing role, as a basis for future discussion and planning for educational needs. This paper describes the role of the genetic nurse within the United Kingdom and offers suggestions for assessment of competency. Strong themes emerging from respondents' comments include the need and desire for multi-professional team work, and it is apparent that most respondents felt the families' needs would best be served by a skilful combination of medical and nursing input, rather than adherence to traditional roles.

$(\mathcal{F}$ Med Genet 1997;34:141-147)
\end{abstract}

Keywords: role of genetic nurse; genetic nurse specialist practice; multi-professional team.

Clinical genetics is acknowledged as a rapidly expanding field within the health services. ${ }^{1}$ The development of new technology, ${ }^{2}$ an increasing consumer awareness of genetics, and the demand by clients for increasing information and testing ${ }^{3}$ have exerted pressure on the National Health Service (NHS) to develop Regional Genetic Services covering the United Kingdom (UK). The delivery of genetic counselling has evolved initially from a medical model, based on the philosophy that people have an inherent right to be properly informed about genetic risks and reproductive options, and that they should be supported during any decision making process. ${ }^{24}$ Genetic counselling is therefore not concerned only with the provision of information, but involves consideration of the psychological impact of that information. The need for a genetic counselling service to include resources to enable clients to explore their feelings in the context of the inherited condition was identified by Fraser $^{5}$ as early as 1974. Nurses and social workers have been gradually integrated into the team since that time, and a report by the Working Party of the Clinical Genetics Society in $1982^{6}$ indicated that the genetic services which employed nursing staff as counsellors valued their contribution.

A description of the role of the genetic nurse in the UK was first published by Farnish ${ }^{7}$ in 1988, when the membership of the Genetic Nurses and Social Worker's Association (GNSWA) was 59. Nurses were reported as undertaking preclinic home visits in the majority of centres, attending clinics, and visiting clients at home after the clinic consultation Teaching of other health professionals by nurses was common, as was involvement in research projects within the field. However, there was no mention in the study of nurse only contacts, other than in the context of using nurses to access clients who failed to attend the clinic.

There are now over 108 members of the Association of Genetic Nurses and Counsellors (formerly the GNSWA) within the UK, the majority of whom are based in regional genetic centres. For the sake of brevity, and to reflect the background of the majority of the membership, the term nurse will be used to describe all genetic coworkers. There has been a powerful medical influence on the practice of genetic nurses, as most are accountable clinically to a consultant in clinical genetics. However, the development of nurse specialist and advanced practitioner status in many specialised fields has also affected the role of the nurse, and nurses are increasingly willing to accept professional responsibility for their work and in certain cases to work autonomously, albeit under the guidance of clinical colleagues. These developments have been the subject of much discussion within regional genetic centres. While in some centres genetic nurse specialists carry their own clinical case load, as well as working with a consultant, the development of such autonomy has been opposed in other centres. During 1994, members of the GNSWA raised these issues as a concern for the profession as a whole, and it was decided to establish a Working Party to investigate the role of the genetic nurse throughout the UK. It was hoped that an initial study would provide a current description of the role of nurses, and would provide a basis for informed discussion and future recommendations with regard to the development of the role and the future education of genetic nurses.

\section{Aims of the study}

The aims of the Working Party study were to describe the current role and practice of the genetic nurse, to ascertain views from both nursing and medical staff on the value of 
Table 1 Categories of client groups

(1) Huntington's disease

(2) Duchenne muscular dystrophy/fragile X

(3) Cystic fibrosis

(4) Chromosomal translocations

(5) Trisomies

(6) Dysmorphology

(7) Multifactorial conditions

(8) Familial cancer

(9) Bereavement

(10) Fetal loss

(11) Current pregnancy

(12) Predictable communication difficulties

different aspects of the role, and to use this information in the future as a basis for discussion about job descriptions and educational needs.

\section{Methodology}

The membership of the Working Party consisted of four experienced genetic nurses from different regional centres within the UK. A questionnaire was devised by the members of the Working Party and comprised three main sections: (1) closed questions to provide a professional profile of the nurse and the tasks regularly performed by that nurse, (2) questions about the frequency of certain activities, (3) requests for open ended commentary to ascertain the views of nurses and doctors on aspects of the nurse's role.

The questionnaire covered possible components of the nursing role such as "preclinic" contact, "postclinic" contact, "nurse only" contact, psychotherapeutic counselling, and the educational role of the nurse. A diary sheet was provided to record time spent on activities over the course of a normal working week. The "nursing" questionnaire was sent to every member of the GNSWA ( $n=108)$. A modified form of the questionnaire (in which the individual nurse profile questions were omitted) was sent to the consultant medical geneticists $(n=65)$ in every regional genetic centre in the UK, to try to obtain the opinion of medical geneticists about the role of nurses within their department, and their thoughts about the future development of the role. No follow up reminders were sent as both nursing and medical questionnaires were returned anonymously. It was felt that anonymity was important in order to ensure the frankness of respondents. The questionnaire responses were collated manually by members of the Working Party. A content analysis of the freehand commentary was undertaken to establish emerging themes from both the nursing and medical respondents.

\section{Results}

In total 98 respondents returned the questionnaire. This comprised 52 medical staff $(80 \%$ of those surveyed) and 46 nursing staff ( $43 \%$ of those surveyed).

PROFILE OF NURSING RESPONDENTS

A total of $91 \%(n=42)$ of nurse respondents were registered nurses, and the majority also had an additional postregistration qualification, such as health visiting $(n=21)$ or mid- wifery $(n=20)$ or both. Only $26 \%(n=12)$ had completed a specific course in genetics; most of those had undertaken the Welsh National Board genetics module (at certificate or diploma level) with which the Cardiff Medical Genetics department is associated, or the short course run at the Institute of Child Health in London. Of particular interest at this time, with the introduction of Post Registration Education and Practice (PREP), ${ }^{8}$ is the finding that $24 \%$ of the nursing respondents have completed or are currently taking postgraduate degrees in either genetics or subjects related to the nursing role, such as health care, health education, or health psychology. The United Kingdom Central Council (UKCC) PREP document recognises that advanced practitioners are likely to be those nurses educated to Masters or PhD level and, therefore, this trend within genetic nursing is consistent with proceeding to specialist or advanced nursing practice. The average length of experience in clinical genetics was 5.6 years (range 7 months to 15 years).

ANALYSIS OF COMPONENTS OF NURSING PRACTICE An analysis of the diary sheets completed by respondents and the questionnaires identified the following tasks undertaken by genetic nurses.

Clinical

(1) Preclinic home visits to take pedigree, obtain information, ascertain family concerns, and give family information about condition and genetic service.

(2) Attendance at clinics.

(3) Postclinic follow up visits, to reinforce information or offer further counselling.

(4) Contact with clients in the home or clinic, who will not see a doctor (nurse only contact), to provide genetic counselling and information.

(5) Obtaining blood samples for genetic studies.

(6) Provision of supportive counselling to clients with specific counselling needs, such as during decision making.

\section{Organisational}

(7) Organisation of prenatal tests.

(8) Preparation for clinics, for example, ordering notes, arranging clinic schedules.

(9) Attending clinical or business meetings, both within and outside the department.

(10) Maintenance of genetic registers.

(11) Administration tasks.

Professional

(12) Supervision and support of other staff.

(13) Education/training necessary for the nurse's own development.

(14) Input into the nurse's own research project or assistance with other's research.

(15) Liaison with other professional groups, such as laboratory or clinical staff in other specialties.

(16) Teaching professionals or other groups. 
Pre-clinic visits

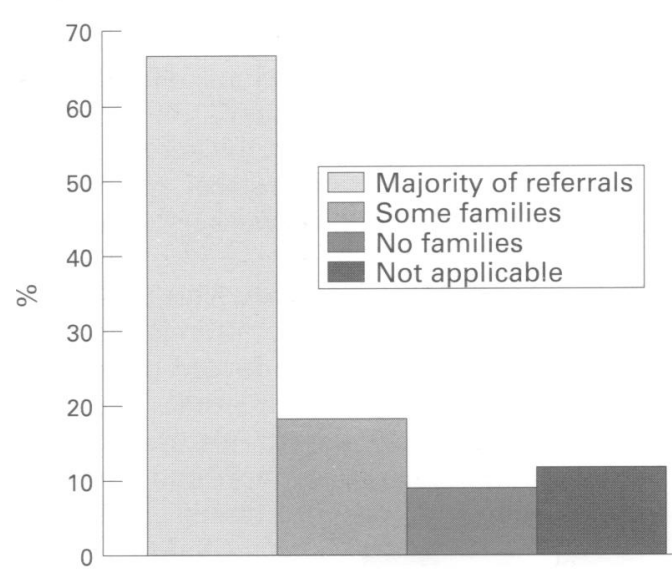

Proportion of pre-clinic visits

Figure 1 Preclinic visits

PRECLINIC VISITS

In many genetic centres, a referred family is seen at home by a genetic nurse before the clinic appointment (preclinic visit). To discover how widespread this practice was, a section of questions in both questionnaires related to preclinic visiting. Of 41 nurses for whom this question was appropriate, 35 replied that preclinic visiting was the norm within their practice (fig 1). The Likert type scale, which was used to ascertain whether preclinic visiting was done in a variety of 12 different client groups (table 1), indicated that where prioritisation occurs, preclinic visiting is generally undertaken in families where there is a known mendelian condition, and less frequently in cases of multifactorial disease or dysmorphic syndromes. This trend was confirmed by responses from medical staff. The value of preclinic visiting was judged by respondents to be (1) increased efficiency of service delivery, (2) preparing the client for the genetic consultation, and (3) delivering psychosocial aspects of care before the clinic consultation. The basis for allocation of high or low priority for preclinic visits in different disease groups is therefore unclear, as presumably these aspects of care would be relevant in most cases.

"Home visit allows family and nurse to assess the need for appropriate timing of appointment. Allows assessment for need to attend clinic at all (depends on expectations of referral). Allows family to prepare, ascertain family information and understand what they may expect - 'setting the scene'." (Nurse)

"It gives us a good idea about how the clinic appointment should be structured and how the family may cope with the information." (Nurse)

"Confirmation of diagnosis and medical details. Assessment of family dynamics and social conditions." (Nurse)

"It is of value in .... both the provision and quality of service. In terms of provision, more patients are likely to attend clinic if they have had contact. Clinics can be better planned. Patients' expectations and their apprehensions can be dealt with. Pedigree information etc obtained which saves time in clinic and, hence,

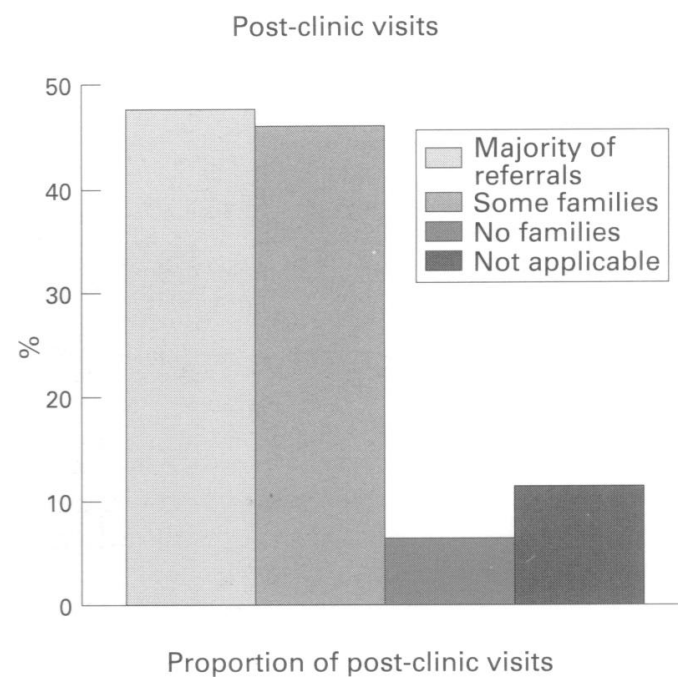

Figure 2 Postclinic visits.

clinic time can be better spent in discussion and examination. Issues for the family can be identified before clinic, family can have thought through their questions before clinic, etc. Permission to obtain case notes obtained etc, not to mention the quality of care and discussion and identification of psychosocial issues which may not come out at clinic." (Doctor)

"I think all families gain from the home visit, due to the contact and a familiar face at the clinic appointment." (Doctor)

\section{POSTCLINIC VISITS}

Replies from nursing respondents indicated that the majority $(n=37)$ are offering postclinic contact (fig 2). This type of contact was aimed primarily at consolidation and reinforcement of information given in clinic, and to assess future needs for the family.

"For the more complicated genetic diseases often the family cannot take it all in at one time. It is often helpful to give them the opportunity to clarify what is said and to discuss options." (Nurse)

"If the family need extra support or information, then a follow up visit is very valuable. This is not dependent on the condition, but is dependent on their individual situation and perception of the condition." (Nurse)

\section{NURSE ONLY CONTACT}

Nurse only contact was defined as contact between the nurse and family, either at home or in a clinic situation, where there was no direct medical input. Again, the majority of nurses (35/41) replied that they held a case load of families who are not seen by one of their medical colleagues, and this trend was reinforced by replies from the medical staff (43/50) (fig 3). Of 12 different client groups, nurses replied that they most frequently saw clients with recessive conditions such as cystic fibrosis (22/46), trisomies (17/46), or neural tube defects (14/46). Neither medical nor nursing staff felt that nurse only contact was appropriate in cases where a dysmorphic syndrome was queried (fig 4). Where medical staff saw an original member of the family, nurses were often 
Nurse only contact

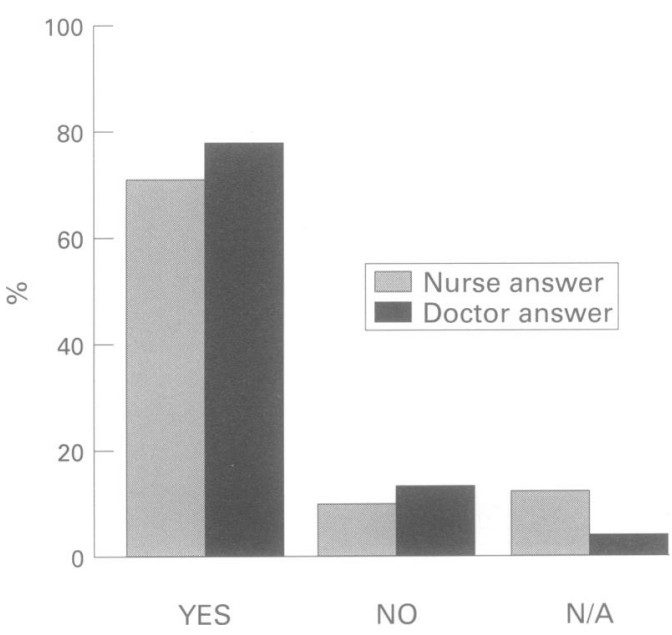

Figure 3 Nurse only contact.

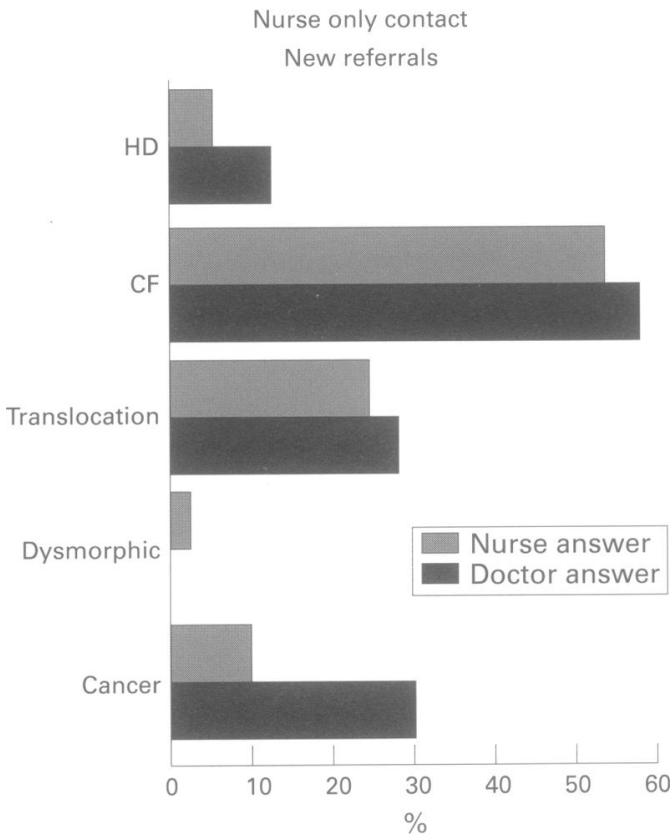

Figure 4 Nurse only contact: new referrals.

responsible for contact with the extended family. This is especially relevant in mendelian conditions or chromosome translocations where the extended family may request carrier testing. The value of nurse only contact was mainly viewed as maximising efficiency, an appropriate use of resources, and an improvement in client care. Three medical staff stated that they felt this method of working was a realisation of the team approach within genetics, and six geneticists considered that this aspect of the nursing role enhanced individual job satisfaction and helped nurses to develop their role.

"There are more nursing hours available than consultant hours; more cost effective. Families often find nurse contact less traumatic." (Doctor)

"We all work in a team and should share work which should be done by whoever is able." (Doctor)

"Increased efficiency of service/skill mix etc and developing role of nurse." (Doctor)
"Good use of resources, and where diagnosis is known, I feel the co-worker is often the best person to spend sufficient time and care in discussion of risks/patient's concerns etc." (Nurse)

"Quicker response to referral, often at home, therefore, reduces waiting list. Satisfying for co-worker." (Nurse)

CLINIC ATTENDANCE BY THE NURSE

The attendance of a genetic nurse at a clinic conducted by medical staff was considered by both nurses and doctors to be valuable, but both groups felt that the value lay in the support and contact which could be offered to the family in the clinic, rather than being of specific value to the department.

\section{PSYCHOTHERAPEUTIC COUNSELLING}

The majority of respondents (78/98) stated that within their centre families or individua subjects were offered further counselling when needed. Further counselling was defined as counselling of a psychotherapeutic nature, above that which would normally be delivered as part of a genetic counselling approach. However, only $35 \%(n=14)$ of nurses, and $8 \%$ $(n=4)$ of doctors who stated that they offered a counselling service as part of their role had undertaken specific training in counselling skills on a dedicated course. The qualifications cited were generally at either certificate or diploma level, implying $90-450$ hours of training over one to three years. This is of concern, as the Counselling and Psychotherapy Central Awarding Body (CPCAB) ${ }^{9}$ recommends that 150 hours of training in counselling is the minimum required for counsellors to achieve competency in working with a client with a current problem. For counsellors seeing clients with a difficult history, further training is recommended. As many clients seen by a genetics service fall into the latter category by the very presence of an inherited condition within the family, the lack or insufficiency of training for nurses working in this area is striking.

EDUCATIONAL RESPONSIBILITIES

Every nurse respondent reported a responsibility for formal teaching of health professionals, both within training programmes (such as the nursing or midwifery degree students) or in the working environment. In addition, nurses were providing education about clinical genetics to other learning groups such as A level students or student social workers. Forty one percent $(n=19)$ of nurses had a recognised teaching certificate.

\section{GENERAL COMMENTS}

A content analysis of the open ended comments provided by both groups of respondents showed the following themes.

(1) Clinical genetics is a field in which the team approach is an effective way of ensuring holistic client care. Comments from both medical and nursing staff emphasised the role of the medical component of the team in making diagnoses, and the role of the nursing com- 
ponent in providing psychological support. However, a consensus of opinion was that the optimal approach is not in strict delineation of roles, but in a sharing of the strengths of each individual member of the team. This is illustrated in the following comment. "In general, nursing input appears to result in a stronger bond between consultand and genetics team. Where nursing input only occurs, a psychological/social approach to problems may predominate. This is sometimes different in its priorities to a more medical model. The medical model tends to be more rigorous (and ?mediocologically robust), the nursing model more empathetic (sic). A combination of both, for most cases, with discussion between professionals should ensure adequate care and patient autonomy." (Doctor)

(2) There is concern among a small minority of medical staff that the psychosocial care of clients may be seen to be a nursing responsibility, resulting in the exclusion of the doctor from those aspects of family care: "I think the nurse must also be aware that the doctor may appreciate close contacts with the family. Caring, counselling and support are not solely nursing issues and concerns." (Doctor)

(3) A small number of nurses expressed concern that their role might be seen as threatening to the medical staff and, therefore, the development of the role within their workplace was severely restricted. This impression was confirmed by the comments of two doctors, one of whom used the phrase: "nursing staff have almost usurped the medical role in clinical genetics."

\section{DEVELOPMENT OF THE ROLE OF THE GENETIC} NURSE

In general, it appeared that nurses were practising in a way which was satisfying and fulfilling to them personally, as well as facilitating the provision of clinical genetic services. This is certainly consistent with the findings of the study by Farnish. ${ }^{7}$ Nurses were not so concerned with developing their role further as they were with ensuring that they had adequate time and resources to fulfil patient needs for contact. For example, $28 \%(n=12)$ felt they could not offer clients sufficient postclinic follow up or supportive counselling when they perceived an actual need. As many as $44 \%$ $(n=19)$ stated they had insufficient time to perform their current roles adequately, the most common reason being inappropriate or inadequate staffing within the department, which meant secretarial or clerical work was being done by nurses.

The role developments which appeared controversial in a few cases were those concerning the adoption of an autonomous case load by nurses ("nurse only" contacts) under the clinical responsibility of a doctor, and the provision of psychosocial support for patients by nurses. However, as has been stated, the great majority of doctors fully supported the contribution of nurses in these areas, and it was suggested by some that, with the increasing numbers of clients seeking genetic counselling because of familial cancer, this was an ideal area for further development of the role.

\section{Discussion}

This study broadly describes the activities of genetic nurses throughout the UK. The responses obtained from the medical questionnaires verified the replies from the nursing questionnaire with regard to current practice of genetic nurses.

The reasons for the disparity in response rate between nursing and medical staff are not obvious. However, a diary section was included in the nursing staff questionnaire and it was thought retrospectively by the Working Party that this may have discouraged nurses from completing the questionnaire, as it added considerably to the effort involved. Another possible explanation is that in the market economy of the NHS at present, it is mainly the geneticist who deals with purchasers, so it could be that justifying employment of nurses in the service has been more of a business issue to the geneticist than to the genetic nurse.

While there are individual differences, the activities of genetic nurses were shown to be perceived by both nursing and medical staff as remarkably similar between different centres. Preclinic visiting and postclinic contact with families was seen to be the norm by the majority of respondents, and was perceived to be of positive benefit to both families using the service, and the efficient and effective running of the service itself. The majority of nurses are carrying individual case loads and have contact with families who are not seen by a geneticist. The comments provided by the respondents indicated some general guidelines which could be followed with regard to nurse only contact. These general guidelines would indicate that nurse only contact is appropriate in families where: (1)The needs of the family are able to be met by this approach. These would include the needs for explanation of an inheritance pattern, discussion about the reproductive options available to a family, and organisation of appropriate genetic testing, but obviously would not include clinical opinion about the course of a condition in an individual person. (2) A definite diagnosis has been established. Nurses are not trained as diagnosticians; therefore clients must see a doctor when there is any uncertainty as to the diagnosis. Further to this point, the nurse must be sufficiently competent to detect the possibility of misdiagnosis and seek guidance from medical staff as appropriate. Wherever possible, documentation of the diagnosis must be pursued. (3) The nurse involved is sufficiently experienced to deal competently with the situation. While competency can be defined in terms of training and experience, in practical terms it is often assessed by observation of skills and performance by colleagues. The skills required within genetics are frequently acquired through observing good practice modelled by others. A system of training, orientation, and assessment whereby nurses are required both to observe others at work and to be observed may be one method of ensuring competency. A key feature 
of competency in a nurse is the ability to assess the need for input from other professionals in the field and to make referrals. Thus, an integral component of competency is an understanding of the limits of one's own practice.

In general terms, families which may fit the criteria for nurse only contact would include those with a family history of recessive conditions, trisomy, neural tube defects, balanced chromosome rearrangements, and consanguinity. In families with dominant or $\mathrm{X}$ linked conditions (such as Duchenne muscular dystrophy) the original proband may be seen by medical staff, but relatives in the extended family may be appropriately seen by a nurse only. The nurse is able to offer information, discussion, and counselling to clients in these groups, frequently in the environment of their own home. The value of the nursing role in seeing these clients may be clarified by the work of Brock, ${ }^{10}$ who is convinced of the therapeutic nature of the opportunity afforded to clients of a genetic service simply to tell their story. This theory may explain the value of genetic counselling to clients, even when medical skills are not required, and it is frequently the genetic nurse who has the time, opportunity, and skills to listen to the narrative. While the ability of many doctors to provide psychological support to clients is undisputed, it is also possible that medical tasks, such as examination and taking a medical history, may actually interfere with the family story telling Brock $^{10}$ describes as helpful to the client.

This study broadly confirms the findings by Farnish $^{7}$ in 1988, who emphasised the advantages to the family of using a team approach in clinical genetics. The description of roles is also consistent with that published by Fibison ${ }^{4}$ in a paper which addressed the role of nurses in provision of genetic services within the United States of America. Within a team, there may be conflict about role boundaries, particularly if professional trust is absent. However, the discussion about role boundaries between medical and nursing staff is not confined to genetics. Salvage ${ }^{11}$ commented recently that the use of expert nurses to complement medical input rather than replace it would improve services to patients in general. This opinion is confirmed by Wilson ${ }^{12}$ and it is to be hoped that studies such as this one will be of interest and benefit to the broader discussion about the roles of both medical and nursing staff in similar health services in the future.

It would appear that to fulfil the roles described by both the nursing and medical respondents to this and other studies, several key competencies for the genetic nurse could be suggested. (1) The ability both to understand and to communicate information concerning the basis of genetic disease, inheritance patterns, and options for testing. (2) The ability to take an accurate family history and to understand the implications of past medical history. (3) The ability to provide a counselling environment in which clients can safely explore the implications of the condition and their own choices. (4) The insight to understand their own capabilities and to seek guidance and refer appropriately where necessary.

This study was undertaken as a basis for further discussion about the educational preparation for genetic nurses. The recommendations for training will need to be made in conjunction with some guidelines for assessing competency. At present, this is done in either an ad hoc fashion within a department, or by using a system such as Individual Performance Review, but clearly more uniform methods would be helpful to both nurses and those assessing them. The Association of Genetic Nurses and Counsellors (AGNC) has already begun to address the issue of competency, standards, and education, using both the results of this work and further work on the skills required for genetic nursing. ${ }^{13}$

\section{Conclusion}

The role and practice of the genetic nurse has been described in terms of activity, and there has been a broad consensus between genetic nurses and their medical colleagues as to the nature and value of the role. However, it is clear that this study has addressed the role of the genetic nurse only in terms of the opinion of members of the clinical genetics team. It has not in any way addressed the effect of the genetic nurse input in terms of the client's perspective. Further studies of the perceived effect of the nurse's role from the client's point of view and how the work of the nurse affects outcomes will need to be undertaken to ascertain whether the role is actually effective.

In addition to client contact, genetic nurses have a role in the education of both professional colleagues and the general community and are involved in research. This basic study will be helpful in determining guidelines for job descriptions of genetic nursing posts in the future, and will help to establish what could be considered as standard practice within the speciality. However, it is clear that further work is needed to determine firm criteria for competency and to set both clinical and educational standards. It may be that many nurses are fulfilling the counselling and teaching aspects of the role, without necessarily having the appropriate training to do so. While competency does not depend solely on formal training, this issue must be addressed by both nurses and those to whom they are clinically responsible. The AGNC is actively exploring standards for competency and educational requirements, and welcomes contributions to the debate.

1 McLean SAM. The new genetics: a challenge to clinical values? Proc $R$ Coll Physicians Edinburgh 1996;26:41-50.

2 Clarke A. Genetics, ethics and audit. Lancet 1990;335:11457.

3 Tymstra TJ. The imperative character of medical technology and the meaning of "anticipated decision regret". Int $\mathcal{F}$ Technol Assess Health Care 1989;5:209-13.

4 Fibison WJ. The nursing role in the delivery of genetic services. Issues in Health Care of Women 1983;4:1-15.

ices. Issues in Health Care of Women 1983;4:1-15. 636-59.

6 Report of the Clinical Genetics Society Working Party on Regional Genetics Services. The provision of regional genetic services in the United Kingdom. Eugen Soc 1982;suppl 4

7 Farnish SA. A developing role in genetic counselling. $f$ Med Genet 1988;25:392-5. 
8 UKCC. PREP and you. London: United Kingdom Central Council for Nursing, Midwifery and Health Visiting, 1995. Council for Nursing, Midwifery and Health Visiting, 1995. Counselling and Psychotherapy Awarding Body. Develop-
ing and assessing counsellor competence. Tutor manual, ing and assessing coun

10 Brock SC. Narrative and medical genetics: on ethics and therapeutics. Qual Health Res 1995;5:150-68.
11 Salvage J. What's happening to nursing? $B M 7$ 1995;311 274-5.

2 Wilson $S$. Report queries savings with doctor-nurse shift BMF 1995;311:280

13 Guilbert P. Defining the skills and knowledge base of genetic nursing practice. BSc dissertation, University of Nottingham, 1966 